\title{
Construção e validação de um instrumento de enfermagem para avaliação da dispneia
}

\author{
Construction and validation of a nursing instrument for the evaluation of dyspnea
}

\section{Construcción y validación de un instrumento de enfermería para laevaluación de ladisnea}

Taís dos Passos Sagica ${ }^{1 *}$, Viviane Albuquerque Farias ${ }^{1}$, Crislen de Melo Conceição ${ }^{1}$, Jean Vitor Silva Ferreira ${ }^{1}$, Amanda Carolina Rozario Pantoja ${ }^{2}$, Vanessa Kelly Cardoso Estumano ${ }^{3}$, Glória Yanne Martins de Oliveira ${ }^{3}$, Clarice Geórgia Monteiro Dias e Silva ${ }^{3}$, Cintia Yolette Urbano Pauxis Aben-Athar ${ }^{1}$, Aline Maria Pereira Cruz Ramos ${ }^{1}$.

\section{RESUMO}

Objetivo: Construir e validar um instrumento de enfermagem para avaliação da dispneia, visando sua potencial aplicação na assistência à saúde. Métodos: Trata-se de um estudo prospectivo, transversal de abordagem quantitativa. Foi construído um instrumento com 13 itens que passou por validação de conteúdo com 12 enfermeiros atuantes na clínica de pneumologia de um Hospital Universitário, mediante avaliação de três variáveis: a clareza, a pertinência e a relevância. A análise estatística ocorreu por meio do Coeficiente de Correlação Intraclasse (ICC) (concordância 80\%) e teste do qui-quadrado. Resultados: Individualmente, as três variáveis apresentaram índices elevados de correlação entre os juízes, os itens "gestão de cuidados" e "ficha de acompanhamento", obtiveram 90,5\% quanto a clareza. O item "sinais de desconforto respiratório" apresentou maior correlação referente a pertinência e a relevância com 93,9\% em ambas. O ICC final do instrumento foi de 0,173 (ótima confiabilidade), com $p$ valor 0.45 . Apenas um item não conseguiu alcançar os valores mínimos estabelecidos, sendo excluído do instrumento final. Conclusão: Esta ferramenta possibilita o norteamento das condutas de enfermagem, auxiliando na tomada de decisão com satisfatório alcance da eficácia, uma vez que, evidencia as dimensões, classificações e influências da dispneia.

Palavras-chave: Dispneia, Enfermagem, Saúde.

\begin{abstract}
Objective: To build and validate a nursing instrument for assessing dyspnea, aiming at its potential application in health care. Methods: This is a prospective, cross-sectional study with a quantitative approach. An instrument with 13 items was constructed, which underwent content validation by 12 nurses working in the pulmonology clinic of a University Hospital, through the assessment of three variables: clarity, relevance and relevance. Statistical analysis was performed using the Intraclass Correlation Coefficient (ICC) (80\% agreement) and the chi-square test. Results: Individually, the three variables showed high levels of correlation between the judges, the items "care management" and "follow-up form", obtained $90.5 \%$ regarding clarity. The item "signs of respiratory distress" showed a greater correlation regarding pertinence and relevance with $93.9 \%$ in both. The final ICC of the instrument was 0.173 (excellent reliability), with $p$ value 0.45 . Only one item failed to reach the minimum values established, being excluded from the final instrument. Conclusion: This tool makes it possible to guide nursing practices, assisting in decision-making with a satisfactory range of effectiveness, since it highlights the dimensions, classifications and influences of dyspnea.
\end{abstract}

Keywords: Dyspnea, Nursing, Health.

1 Universidade Federal do Pará (UFPA), Belém - PA. *E-mail: thaispassos12@gmail.com

2 Universidade da Amazônia (UNAMA), Belém - PA.

${ }^{3}$ Hospital Universitário João de Barros Barreto (HUJBB). Belém - PA.

SUBMETIDO EM: 6/2020

ACEITO EM: 7/2020

PUBLICADO EM: 9/2020 


\section{RESUMEN}

Objetivo: construir y validar un instrumento de enfermería para evaluarladisnea, conel objetivo de suposibleaplicación en laatención de lasalud. Métodos: Este es un estudio prospectivo, transversal con un enfoque cuantitativo. Se construyó un instrumento con 13 ítems, que fue sometido a validación de contenido por 12 enfermeras que trabajan en la clínica de neumología de un Hospital Universitario, a través de laevaluación de tresvariables: claridad, relevancia y relevancia. El análisis estadístico se realizó utilizando el coeficiente de correlaciónintraclase (ICC) (acuerdodel 80\%) y laprueba de chi-cuadrado. Resultados: Individualmente, lastresvariablesmostraron altos niveles de correlación entre losjueces, losítems "gestión de laatención" y "formulario de seguimiento", obtuvieron 90.5\% conrespecto a laclaridad. El ítem "signos de dificultadrespiratoria" mostró una mayorcorrelaciónconrespecto a lapertinencia y relevanciacon $93.9 \%$ en ambos. El ICC final del instrumento fue de 0.173 (excelente confiabilidad), con un valor de p de 0.45 . Solo un ítem no alcanzólos valores mínimos establecidos, quedando excluidodel instrumento final. Conclusión: esta herramienta permite guiar lasprácticas de enfermería, ayudando en la toma de decisionescon un rango satisfactorio de efectividad, ya que destaca las dimensiones, clasificaciones e influencias de ladisnea.

Palabras clave: Disnea, Enfermería, Salud.

\section{INTRODUÇÃO}

A dispneia é definida como uma experiência subjetiva de desconforto respiratório que varia em intensidade. Esta é multifatorial e sofre influência fisiológica, psicológica, social e ambiental, o que induz a respostas fisiológicas e comportamentais secundárias (GENTZLER ER, et al., 2018). Com fisiopatologia complexa que surge com comprometimento e alerta para a possibilidade de ameaça de homeostase, a dispneia envolve a ativação de vias que levam ao aumento do trabalho respiratório, a estimulação dos receptores das vias aéreas, parênquima pulmonar ou parede torácica, além da estimulação excessiva do centro respiratório pelas artérias central e quimiorreceptores periféricos (ANZUETO A e MIRAVITLLES M, 2017).

De origem respiratória ou cardíaca, quase $90 \%$ dos casos de dispneia são acometidos por asma, insuficiência cardíaca, isquemia miocárdica, Doença Pulmonar Obstrutiva Crônica (DPOC), pneumonia e distúrbios psicogênicos, podendo interferir no desempenho das Atividades de Vida Diária (AVD) ocasionando mudanças no estilo de vida (COCCIA CBI, et al., 2016). Desta forma, caracterizada como um sintoma frequente e desestabilizador em pacientes com doenças crônicas, progressivas e incuráveis em sua variedade clínica, a dispneia pode aumentar sua intensidade e prevalência ao longo da vida, por esse motivo torna-se imprescindível detectar sinais de alerta que exijam atenção imediata, pois é um sintoma angustiante, não só para o enfermo, como também para família e profissionais da saúde (COCCIA CBI, et al., 2016; ALVES ARMV e PINA PSRS, 2018; GENTZLER ER, et al., 2018).

Por se tratar de um desconforto subjetivo, a caracterização do sintoma, na maioria dos casos é realizada pelo próprio doente, o mesmo estando em posição de determinar o grau de intensidade e severidade (GENTZLER ER, et al., 2018).

Em um estudo realizado com 77 pacientes, buscou-se comparar os registros de enfermagem e a autoavaliação da intensidade da dispneia tendo como recurso a Escala de Avaliação Numérica (EAN) aplicada em indivíduos com doenças crônicas, progressivas e avançadas. Neste estudo, somente $38 \%$ da amostra apresentou diagnóstico de dispneia em repouso e/ou dispneia funcional. Neste sentido, as intervenções realizadas foram a monitorização da frequência respiratória, otimização da respiração por meio de técnicas de posicionamento no leito, utilização de cinesioterapia respiratória e oxigenioterapia (ALVES ARMV e PINA PSRS, 2018).

O reconhecimento dos três domínios principais: (i) experiência sensorial do paciente, (ii) angústia respiratória e (iii) impacto dos sintomas / doenças; possibilita traçar planos de cuidados visando melhoria clínica em tempo hábil (DANGERS L, et al., 2017; DUNCAN D e ASHBY A, 2018; HANANIA NA e O'DONNELL DE, 2019; DAMANI A, et al., 2018). 
Assim, a avaliação da dispneia permeia o uso de escalas de avaliação que complementam os achados clínicos da ausculta pulmonar realizada no exame físico do paciente (HASHIMOTO H e KANDA K, 2019; ANZUETO A e MIRAVITLLES M, 2017; HANANIA NA e O'DONNELL DE, 2019).Todavia, a maioria delas aborda essa dificuldade respiratória de modo unidimensional, com ênfase em uma patologia específica (CRUZ A, et al., 2017).

Por prestarem assistência ininterrupta, os enfermeiros devem estar habilitados a avaliar de forma precisa as alterações cognitivas e fisiológicas que configuram a dispneia em âmbito multidimensional, considerando também a história clínica do paciente (DANGERS L, et al., 2017; DUNCAN D e ASHBY A, 2018; HANANIA NA e O'DONNELL DE, 2019). Neste contexto, o uso de ferramentas de avaliação da dispnéia é fundamental para a equipe de enfermagem durante a assistência, proporcionando o reconhecimento precoce nas alterações do padrão respiratório, evitando assim agravos e, consequentemente, um melhor direcionamento no cuidado (JELLINGTON MO, et al., 2016).

Sendo assim, este estudo objetiva construir e validar um instrumento de enfermagem para avaliação da dispneia, visando sua potencial aplicação na assistência à saúde.

\section{MÉTODOS}

Trata-se de um estudo prospectivo, transversal de abordagem quantitativa. Este constituiu-se em duas fases: a construção e a validação de conteúdo de um instrumento de enfermagem para avaliação da dispneia. Foi desenvolvido no setor de pneumologia de um Hospital Universitário Federal, no período de agosto a dezembro de 2018.

Inicialmente, realizou-se um processo de investigação da literatura disponível sobre a temática, sendo utilizadas as bases de dados indexadas na Biblioteca Virtual em Saúde (BVS), em especial Medical Literature Analysis and Retrievel System Online (MEDLINE). Tendo como principais descritores: dispneia e enfermagem.

Após a análise da literatura, foram levantados os itens do instrumento, constituindo-se de 13 , sendo estes: identificação do paciente; denominações; condições associadas ao surgimento da dispneia; história da dispneia; história clínica; dispneia para o usuário; fatores que desencadeiam a dispneia; escalas de avaliação; sinais de desconforto respiratório; classificação quanto ao ritmo; plano de cuidados; gestão de cuidados e ficha de acompanhamento.

Foram convidados especialistaspara o processo de validação do conteúdo segundo os critérios de inclusão: profissionais com graduação e especialização em Enfermagem; atuantes na clínica de Pneumologia do local de estudo; que aceitaram participar mediante a assinatura do Termo de consentimento Livre e Esclarecido (TCLE). Do total de 13 enfermeiros elegíveis, 12 profissionais aceitaram participar. Estes avaliaram e responderam ao questionário estruturado com base no estudo de Magalhães (2007) e adaptado aos critérios da pesquisa. Cada item foi avaliado considerando três variáveis: a clareza, a pertinência e a relevância, cujo conceito atribuído poderia ser: insuficiente (0), bom (1), regular (2) ou excelente (3).

A análise estatística ocorreu por meio do Coeficiente de Correlação Intraclasse (ICC) sendo este uma das ferramentas mais utilizadas para determinar a confiabilidade de medidas. Ele é calculado como a proporção da variabilidade atribuída aos objetos em estudo (razão entre esta variabilidade e a variabilidade total, incluindo erro de medição associado) (SHROUT PE e FLEISS JL, 1979). Neste estudo, o cálculo do ICC foi baseado nos modelos de análise variância (ANOVA) de medidas repetidas e calculado a partir de estimativas dos diferentes componentes de variância, através da decomposição da variância total nas variâncias entre sujeitos e dentro dos sujeitos. Foi utilizado o software $R$ versão 4.0.0.

Os parâmetros de análise foram baseados no pressuposto de que o ICC teria ótima confiabilidade (ICC >0,9), boa confiabilidade $(0,7<$ ICC $<0,8)$ e fraca confiabilidade (ICC $<0,6)$. Considerou-se 0 percentual de concordância de $80 \%$ como critério de decisão sobre a permanência do item no instrumento. Adotou-se o Teste de Qui-quadrado com p-valor <0,05. 
O estudo foi desenvolvido segundo os preceitos éticos do Conselho Nacional de Saúde (CNS) respeitando as resoluções $\mathrm{N}^{\circ} 466 / 2012$; $\mathrm{N}^{\circ} 510 / 2016$ e $\mathrm{N}^{\circ} 580 / 2018$, sendo aprovado segundo o parecer $\mathrm{n}^{\circ}$ 2.840.921 do Comitê de Ética em Pesquisa do Núcleo de Pesquisas em Oncologia da Universidade Federal do Pará.

\section{RESULTADOS}

A validação de conteúdo contou com 12 avaliadores, sendo que $9(75 \%)$ eram do sexo feminino e 3 (25\%) do sexo masculino. Quanto a titulação, 1 (8\%) obtinha doutorado, $3(25 \%)$ eram mestres e 8 (67\%) especialistas.

Individualmente, as três variáveis (relevância, pertinência e clareza) apresentaram índices elevados de correlação entre os juízes, com destaque aos itens "gestão de cuidados" e "ficha de acompanhamento", com 90,5\% quanto a clareza. Observa-se que o item "sinais de desconforto respiratório" apresentou maior correlação referente a pertinência e a relevância com 93,9\% em ambas (Tabela 1).

Tabela 1 - Distribuição dos itens do instrumento, de acordo com o percentual de concordância estatística.

\begin{tabular}{ccccccc}
\hline Itens & $\begin{array}{c}\mathbf{C L}^{*} \\
\%\end{array}$ & $\begin{array}{c}\text { Clareza } \\
\text { ICC (95\%) }\end{array}$ & $\begin{array}{c}\mathbf{P P}^{*} \\
\%\end{array}$ & $\begin{array}{c}\text { Pertinência } \\
\text { ICC (95\%) }\end{array}$ & $\begin{array}{c}\mathbf{R T}^{*} \\
\%\end{array}$ & $\begin{array}{c}\text { Relevância } \\
\text { ICC (95\%) }\end{array}$ \\
\hline Identificação & 85.5 & {$[0.04 ; 0.89]$} & 90.2 & {$[0.08 ; 0.93]$} & 90.2 & {$[0.08 ; 0.93]$} \\
Denominações & 78.2 & {$[-0.13 ; 0.85]$} & 90.2 & {$[0.08 ; 0.93]$} & 88.7 & {$[0.06 ; 0.91]$} \\
$\begin{array}{c}\text { Condições Associadas ao } \\
\text { Surgimento da Dispneia }\end{array}$ & 77.6 & {$[-0.22 ; 0.83]$} & 87.1 & {$[0.05 ; 0.90]$} & 83.5 & {$[-0.02 ; 0.87]$} \\
$\quad$ História da Dispneia & 91.1 & {$[0.12 ; 0.94]$} & 93.6 & {$[0.16 ; 0.95]$} & 91.8 & {$[0.13 ; 0.94]$} \\
$\quad$ História Clínica & 88.7 & {$[0.06 ; 0.91]$} & 83.5 & {$[-0.02 ; 0.87]$} & 83.5 & {$[-0.02 ; 0.87]$} \\
Dispneia Para o Usuário & 76.9 & {$[-0.23 ; 0.82]$} & 70.7 & {$[-0.29 ; 0.78]$} & 76.9 & {$[-0.23 ; 0.82]$} \\
Fatores que Desencadeiam a & 90.2 & {$[0.08 ; 0.93]$} & 83.5 & {$[-0.02 ; 0.87]$} & 83.5 & {$[-0.02 ; 0.87]$} \\
$\quad$ Dispneia & & & & & & \\
Escala de Avaliação & 90.2 & {$[0.08 ; 0.93]$} & 88.7 & {$[0.06 ; 0.91]$} & 91.1 & {$[0.12 ; 0.94]$} \\
Sinais de Desconforto & 89.3 & {$[0.07 ; 0.92]$} & 93.9 & {$[0.17 ; 0.95]$} & 93.9 & {$[0.17 ; 0.95]$} \\
$\quad$ Respiratório & & & & & & \\
Classificação Quanto ao Ritmo & 83.5 & {$[-0.02 ; 0.87]$} & 76.9 & {$[-0.23 ; 0.82]$} & 88.7 & {$[0.06 ; 0.91]$} \\
Plano de Cuidados & 83.5 & {$[-0.02 ; 0.87]$} & 87.1 & {$[0.05 ; 0.90]$} & 90.2 & {$[0.08 ; 0.93]$} \\
Gestão de Cuidados & 90.5 & {$[0.09 ; 0.92]$} & 87.1 & {$[0.05 ; 0.90]$} & 83.5 & {$[-0.02 ; 0.87]$} \\
Ficha de Acompanhamento & 90.5 & {$[0.09 ; 0.92]$} & 76.9 & {$[-0.23 ; 0.82]$} & 83.5 & {$[-0.02 ; 0.87]$} \\
\hline
\end{tabular}

Legenda: *CL: clareza; PP: pertinência; RT: relevância.

Fonte: Sagica TP, et al., 2020.

O Coeficiente de Correlação Intraclasse final foi de 0.173 (ótima confiabilidade), com p-valor 0.45 . Considerando-se a clareza, pertinência e relevância, o item "história da dispneia", apresentou o resultado mais satisfatório, com 91.8 (Tabela 2). Conforme o exposto, apenas o item "dispneia para o usuário" possui ICC menor de $80 \%$ sendo assim, excluído do instrumento final validado. Desse modo, o instrumento final consolidou-se com 12 itens, sendo disposto em Dados Suplementar. 
Tabela 2 - Índice de Correlação Interclasse final dos itens do instrumento.

\begin{tabular}{lcc}
\hline Itens & ICC & Valor de p \\
\hline Identificação & 87.4 & 0.71 \\
Denominações & 83.2 & 0.64 \\
Condições Associadas ao Surgimento da Dispneia & 80.3 & 0.53 \\
História da Dispneia & 91.8 & 0.75 \\
História Clínica & 84.6 & 0.66 \\
Dispneia Para o Usuário & 73.3 & 0.31 \\
Fatores que Desencadeiam a Dispneia & 84.9 & 0.65 \\
Escala de Avaliação & 89.8 & 0.73 \\
Sinais de Desconforto Respiratório & 91.6 & 0.76 \\
Classificação Quanto ao Ritmo & 82.4 & 0.59 \\
Plano de Cuidados & 85.7 & 0.69 \\
Gestão de Cuidados & 86.3 & 0.7 \\
Ficha de Acompanhamento & 82.1 & 0.56 \\
\hline Geral & 0.173 & 0.45 \\
\hline
\end{tabular}

Legenda: Teste de Qui-quadrado.

Fonte: Sagica TP, et al., 2020.

O primeiro item do instrumento contempla a identificação do paciente, no que se refere a idade e o diagnóstico de base. No segundo apresentam-se as denominações da dispneia, a qual pode ser classificada em: ortopneia, dispneia de esforço, dispneia paroxistica noturna, flexopneia, asma cardíaca, platipneia ou trepopneia. $O$ item 3 trata-se das condições associadas ao surgimento da dispneia; que podem variar desde a ansiedade a obesidade do paciente.

A história da dispneia é descrita no item 4 que correlaciona o desconforto respiratório no ambiente de trabalho e domiciliar. Já a descrição da história clínica detalhada é exposta no item seguinte o qual aborda a data de surgimento do sintoma, o tipo de instalação (súbita ou progressiva), duração, fatores desencadeantes, além de uma comparação subjetiva do usuário, o número de crises e os fatores acompanhantes e que melhoram a dispneia. Ademais, há um item em que podem ser demarcados os fatores que desencadeiam a dispneia.

No instrumento são utilizadas duas escalas de avaliação sendo estas a Escala de Avaliação Numérica (EAN) e a Medical Research Council (MRC) modificada. Os sinais de desconforto respiratório são abordados em um item específico, tais como a cianose, batimento de asas do nariz, cansaço e/ou agitação, entre outros. Bem como a classificação quanto ao ritmo respiratório, sendo estes: taquipneia, dispnéia suspirosa, ritmo de Biot, ritmo de Cantani, ritmo de Kussmaul, ritmo de Cheyne- stokes, bradpneia e hiperpneia.

O item plano de cuidados subdivide-se em diagnósticos e intervenções de enfermagem específicos para dispneia, podendo ser complementados. $O$ item gestão de cuidados trata-se de um check-list voltado ao gerenciamento das ações de enfermagem. Por fim, tem-se um modelo de ficha de acompanhamento para avaliar continuamente os parâmetros principais da dispneia.

\section{DISCUSSÃO}

A enfermagem tem papel fundamental na avaliação do paciente com dispneia, uma vez que sua assistência é direta e ininterrupta ao paciente. Nesse sentido, a incorporação de um plano de manejo individualizado viabiliza os cuidados não só da enfermagem como também da equipe multidisciplinar, o que pode ser operacionalizado a partir de instrumentos de avaliação e que subsidiem esse acompanhamento (DUNCAN D e ASHBY A, 2018). 
Resultados obtidos em estudos realizados por Baker KM, et al. (2020) e Banzett RB e O'Donnell CR (2014) corroboram os resultados positivos dessa intervenção, nos quais a maioria dos enfermeiros concordou que a avaliação de rotina é essencialmente eficaz, pois subsidia rastreamento do progresso da doença, eficácia do tratamento e prognóstico. Com isso, as ferramentas de avaliação são fundamentais para uma intervenção eficaz e oportuna, uma vez que há subjetividade na avaliação do enfermeiro o que aumenta o risco de desfechos não favoráveis (HASHIMOTO H e KANDA K, 2019).

Dessa forma, são necessários instrumentos bem desenvolvidos e clinicamente úteis de fácil uso e que não interfiram no fluxo de trabalho, para que sejam padronizados institucionalmente (ZHUANG Q, et al., 2019; JELLINGTON MO, et al., 2016).

Assim, avaliar a dispneia é o primeiro passo para gerenciá-la de forma oportuna, ressalta-se que apesar de sua prevalência e gravidade, ainda é frequentemente negligenciada. Ademais a escolha de uma ferramenta de avaliação da dispneia pode ser difícil, não apenas por sua natureza multifatorial, mas também devido a existência de inúmeras ferramentas disponíveis e divergentes, sendo nenhuma direcionada a nossa classe (BANZETT RB e O'DONNELL CR, 2014; DUNCAN D e ASHBY A, 2018).

O instrumento avaliado neste estudo fornece uma análise global do paciente, pois não somente classifica a dispneia, como também considera seu histórico e condição clínica. Além disso, a utilização de um instrumento pode aprimorar a relação profissional-paciente, possibilitando maior eficiência no atendimento e assistência prestada (JELLINGTON MO, et al., 2016).

Neste interim, a análise do presente instrumento por meio do Coeficiente de Correlação Intraclasse final demonstrou ótima confiabilidade. Semelhante ao estudo de Zhuang Q, et al. (2019), no qual a Escala de Observação do Desconforto Respiratório (RDOS) foi utilizada para verificação da dispneia em um estudo com 122 pacientes em cuidados paliativos em um Hospital Terciário em Cingapura, apresentando boa confiabilidade interexaminadores, com uma correlação intraclasse de 0.947 (IC95\% 0.919-0.976). Nessa perspectiva, entre os itens que compõe o presente instrumento ressalta-se a história clínica da dispneia, a qual é crucial para observar seu desencadeamento, evolução ou regresso. Sendo assim, garante maior conhecimento do paciente e profissional para a tomada de decisões, uma vez que evidencia seu início, duração e fatores ambientais influenciadores como atividades diárias. Desta forma, oferta maior subsídio para enquadrar a gravidade da condição atual do paciente (DUNCAN D e ASHBY A, 2018).

Outro fator evidenciado trata-se do plano de cuidados, haja vista que a gestão não individualizada implica em resultados insatisfatórios assim como nos estudos de Alves ARMV e Pina PSRS (2018), em que a ausência de um plano de cuidados personalizado repercutiu em intervenções de enfermagem equivalentes para situações discrepantes, além de não se ter uma avaliação eficaz das ações implementadas. Logo, corrobora-se para a importância da classificação da dispneia, implementação de gestão e plano de cuidados, uma vez que avaliação precoce e frequente torna possível a diferenciação da intensidade, identificação de fatores desencadeadores, de exacerbação e alívio, os quais são aspectos importantes para a adequação de intervenções específicas (ALVES ARMV e PINA PSRS, 2018).

Além disso, o emprego frequente do diagnóstico de enfermagem, item contido na ferramenta, auxilia o processo de tomada de decisão ao viabilizar a assistência sistemática, dinâmica e humanizada, garantindo deliberações menos indutivas e mais científicas (SANTOS BL, et al., 2016).

Assim como nos estudos de Santos NA, et al. (2015) em que se ratifica a importância do conhecimento do processo de enfermagem no contexto das doenças respiratórias, tendo em vista a escassez de ferramentas que o incluem, bem como para conhecer o perfil dos pacientes para direcionamento da assistência.

Reitera-se que a comparação com outros estudos do qual também validam instrumentos para avaliação da dispneia é de grande relevância, pois reverbera a clareza, coesão e objetividade do presente estudo, baseado em evidências científicas. Sendo assim, o instrumento também conta com escalas já validadas, como forma de garantir maior evidência na avaliação. Uma dessas é a Medical Research Council modificada (mMRC) publicada pela American Thoracic Society (ATS), a qual foi desenvolvida para avaliar 
as limitações nas atividades de vida diária de indivíduos com doenças pulmonares. É composta por cinco itens que auxiliam o paciente a relatar seu grau subjetivo de dispneia, escolhendo um valor entre zero e quatro, sendo quatro a pior sensação (BANZETT RB, et al., 2015).

Em um estudo realizado no Japão, em uma amostra de 42 pacientes com DPOC, para avaliação da dispneia foi aplicado a escala mMRC em conjunto com tomografia computadorizada (TC) tridimensional. No presente estudo, avaliou-se a associação de estruturas morfológicas das vias aéreas e dispneia em pacientes com DPOC. Os achados sugeriram que o espessamento da parede das vias aéreas avaliado por TC tridimensional e a escala mMRC poderia ser usado como uma ferramenta de avaliação da dispneia em pacientes com DPOC (YASUI H, et al., 2019).

Outra escala acrescida no instrumento foi Escala Numérica, no qual o paciente relata o desconforto de 0 a 10. Alves ARMV e Pina PSRS (2018) em sua pesquisa realizada em Portugal, fizeram a comparação dos registros de enfermagem e a auto avaliação da intensidade da dispneia realizada com recurso à Escala de Avaliação Numérica (EAN) por indivíduos com doenças crónicas, progressivas e avançadas, com necessidades paliativas, internados em serviços de medicina interna. A análise dos registros permitiu constatar a inexistência de um plano terapêutico diferenciado para o controle sintomático de distintas intensidades da dispneia, principalmente por não haver instrumento que promovesse maior importância da sintomatologia.

É válido ressaltar que a aplicação de escalas não é restrita ao âmbito intra-hospitalar, mas aplicável também no âmbito pré-hospitalar. Lindskou TA, et al. (2020) investigaram a viabilidade do uso de um escore de dispneia subjetivo na ambulância pelos pacientes, com score verbal de 0 a 10. Identificou-se que a dispneia aguda pontuada pelos pacientes era alta, mas diminuía antes da chegada ao hospital, sugerindo alívio dos sintomas conforme sensação de segurança pela assistência prestada.

É importante frisar que o uso de instrumentos para avaliação de dispneia, e principalmente sua adequação ao contexto em que é aplicado torna a qualidade da assistência mais direcional. Como citado no estudo multicêntrico de Hashimoto H e Kanda K (2019) no qual desenvolve-se uma escala de avaliação abrangente para medir a dispneia relacionada ao câncer. Com uma amostra de 239 pacientes japoneses com câncer e dispneia, os resultados apontaram um coeficiente $\alpha$ de Cronbach da escala foi de 0,952 neste estudo e, portanto, um nível aceitável de confiabilidade.

Ressalta-se que a avaliação, gerência e intervenção da dispneia são negligenciadas. Não obstante, sua fisiopatologia e associação com as doenças de base demonstrem importante fator de alerta. Em um estudo com 138 pacientes internados em uma Unidade de Terapia Intensiva (UTI), enfermeiros e cuidadores pessoais de pacientes à beira do leito relataram a percepção dos sintomas de dispneia relacionados à dor, através da escala de classificação numérica. A dispneia foi relatada com a mesma frequência que a dor entre os pacientes. Além disso, identificou-se que os cuidadores pessoais tiveram boa concordância com relatos de pacientes com dispneia moderada a grave. No entanto, mesmo quando detectada pelos enfermeiros, a dispneia parecia estar subnotificada (GENTZLER ER, et al., 2018).

A aplicabilidade do presente instrumento, construído e validado, poderá dar suporte à equipe de enfermagem na prática diária, como uma valiosa ferramenta para avaliar de forma abrangente e multidimensional a dispneia. Ademais, garante maior autonomia dos cuidados de enfermagem, bem como auxilia na comunicação interprofissional, essencial para os direcionamentos dos cuidados ao paciente (PEDUZZI M, et al., 2020). Com relação às limitações deste estudo, evidenciou-se (i) demora na devolução dos questionários pelos avaliadores possivelmente relacionada a sobrecarga de serviço, (ii) ausência de $p$ valor significativo associado ao $\mathrm{n}$ amostral limitado, uma vez que, o foco da abordagem de enfermeiros de uma única clínica. Em contrapartida, dentre os benefícios é possível destacar a aceitabilidade e o envolvimento dos enfermeiros na avaliação do instrumento e as contribuições significativas para o aprimoramento do mesmo. Espera-se que este estudo contribua significativamente para a implementação de rotinas de avaliação constante da dispneia baseadas em evidências cientificas, de forma que motivem estudos adicionais para novos questionamentos sobre a identificação precoce, avaliação e intervenção oportuna da dispneia, a fim de incrementar o cuidado prestado ao paciente nessa condição. 


\section{CONSIDERAÇÕES FINAIS}

O Instrumento de avaliação da dispneia garantiu validade no que tange a clareza, pertinência e relevância, corroborando através de testes estatísticos sua confiabilidade. Esta ferramenta possibilita o norteamento das condutas de enfermagem quanto a obtenção de informações precisamente relevantes, considerando, dessa forma, as reais necessidades do usuário. Ele auxilia ainda na tomada de decisão gerencial com satisfatório alcance da eficácia na gestão do cuidado, uma vez que, evidencia as dimensões, classificações e influências da dispneia. Ressalta-se que este instrumento é uma tecnologia passível de ser reproduzida e implementada com acréscimos satisfatórios na assistência à saúde.

\section{REFERÊNCIAS}

1. ALVES ARMV, PINA PSRS. Dispneia em cuidados paliativos: registos de enfermagem e a autoavaliação da dispneia. Revista de Enfermagem Referência, 2018; serlV(16): 53-62.

2. ANZUETO A, MIRAVITLLES M. Pathophysiology of dyspnea in COPD. Postgraduate Medicine, $2017 ; 129$ (3): $366-$ 374.

3. BAKER KM, et al. Intensive care nurses' perceptions of routine dyspnea assessment. American Journal of Critical Care, 2020; 29 (2):132-139.

4. BANZETT RB, et al. Multidimensional Dyspnea Profile: An Instrument for Clinical and Laboratory Research. The European Respiratory Journal, 2015; 45 (6):1681-1691.

5. BANZETT RB, O'DONNELL CR. Should we measure dyspnea in everyone? European Respiratory Journal, 43 (6): 1547-1550.

6. COCCIA CBI, et al. Dyspnoea: Pathophysiology and a clinical approach. SAMJ: South African Medical Journal, 2016; 106 (1): 32-36.

7. CRUZ A, et al. Instrumentos de avaliação da dispneia e tosse em Cuidados Paliativos: Revisão Sistemática da Literatura. Cuidados paliativos, 2017; 4 (1): 50 - 64.

8. DAMANI A, et al. Prevalence and intensity of dyspnea in advanced cancer and its impact on quality of life. Indian Journal of Palliative Care, 2018; 24 (1): 44-50.

9. DANGERS $L$, et al. Relieving dyspnoea by non-invasive ventilation decreases pain thresholds in amyotrophic lateral sclerosis. Thorax, 2017; 72 (3): 230-235.

10. DUNCAN D, ASHBY A. Managing Chronic Breathlessness in the Community. British Journal of Community Nursing, 2018; 23 (7): 318-321.

11. FUSI-SCHMIDHAUSER T, et al. Conservative Management of COVID-19 Patients-Emergency Palliative Care in Action. J Pain Symptom Manage, 2020; S0885-3924(20)30183-4.

12. GENTZLER ER, et al. Under detection and undertreatment of dyspnea in critically ill patients. Am J Respir Crit Care Med, 2018; 199 (11): 1377-1384.

13. HANANIA NA, O'DONNELL DE. Activity-related dyspnea in chronic obstructive pulmonary disease: Physical and psychological consequences, unmet needs, and future directions. International Journal of Chronic Obstructive Pulmonary Disease, 2019; 14:1127-1138.

14. HASHIMOTO H, KANDA K. Development and validation of the Total Dyspnea Scale for Cancer Patients. European Journal of Oncology Nursing, 2019; 41: 120-125.

15. JELLINGTON MO, et al. Nursing challenges in the relief of dyspnea experienced by COPD patients during hospitalization, 2018; 5 (3): 292-299.

16. LINDSKOU TA, et al. Patient experience of severe acute dyspnoea and relief during treatment in ambulances: a prospective observational study. Scand J Trauma ResuscEmerg Med. 2020; 28(1):24.

17. MAGALHÃES MG. Elaboração e validação de um instrumento de avaliação da qualidade da assistência para hospitais pediátricos. Dissertação (Mestrado Profissional em Saúde da Criança e do Adolescente). UniversidadeEstadual do Ceará. Fortaleza, 2007.

18. O'DONNELL DE, et al. Unraveling the causes of unexplained dyspnea: The value of exercise testing. Clinics in Chest Medicine, 2019; 40(2):471-499.

19. PEDUZZI M, et al. Trabalho em equipe: uma revisita ao conceito e a seus desdobramentos no trabalho interprofessional. Trab. Educ. Saúde, Rio de Janeiro, 2020;18(s1).

20. SANTOS NA, et al. Profile of nursing diagnoses in patients with respiratory disorders. Invest Educ Enferm, 2015; 33(1): 112-118.

21. SANTOS BL, et al. Assistência de enfermagem ao adulto com agravos respiratórios. Revista Recien. 2016; 6(18):85-91.

22. SHROUT PE, FLEISS, JL. Intraclass correlations: Uses in assessing rater reliability. Psychological Bulletin, 1979; 86(2): 420-428.

23. YASUI $\mathrm{H}$, et al. Correlation of the modified Medical Research Council dyspnea scale with airway structure assessed by three-dimensional CT in patients with chronic obstructive pulmonary disease. Respiratory Medicine 146 (2019) 76-80.

24. ZHUANG Q, et al. Validity, Reliability, and Diagnostic Accuracy of the Respiratory Distress Observation Scale for Assessment of Dyspnea in Adult Palliative Care Patients. J Pain Symptom Manage. 2019; 57(2):304-310. 